\title{
一种传输控制协议中的主动拥塞控制机制
}

\author{
徐昌䖌 ${ }^{1,2+}$, 鲜永菊 ${ }^{1,2}$, 唐朝伟 ${ }^{2}$, 杨士中 2 \\ ${ }^{1}$ (重庆邮电大学 通信与信息工程学院,重庆 400065) \\ ²(重庆大学 通信工程学院,重庆 400030)
}

\section{An Active Congestion Control Mechanism for Transmission Control Protocol}

\author{
XU Chang-Biao ${ }^{1,2+}, \quad$ XIAN Yong-Ju ${ }^{1,2}, \quad$ TANG Chao-Wei ${ }^{2}, \quad$ YANG Shi-Zhong $^{2}$ \\ ${ }^{1}$ (College of Communication and Information Engineering, Chongqing University of Posts and Telecommunications, Chongqing 400065, \\ China) \\ ${ }^{2}$ (College of Communication Engineering, Chongqing University, Chongqing 400030, China) \\ + Corresponding author: E-mail: xucb@cqupt.edu.cn
}

Xu CB, Xian YJ, Tang CW, Yang SZ. An active congestion control mechanism for transmission control protocol. Journal of Software, 2008,19(6):1533-1545. http://www.jos.org.cn/1000-9825/19/1533.htm

Abstract: To improve performance of TCP (transmission control protocol) Reno, a widely used TCP version, an improved TCP Reno named DAA-TCP is proposed. DAA-TCP (dual AMID-based active TCP) combines dual AIMD (additive-increase multiplicative-decrease) algorithms and adds an active congestion control manner, i.e., the congestion window may be decreased when some given conditions are satisfied before the congestion really occurs. DAA-TCP performances are investigated through simulations. Comparing with TCP Reno, the throughput may be improved; the retransmitted packets and the retransmit probability may be decreased. DAA-TCP can coexist with TCP Reno very friendly. In addition, DAA-TCP is obtained through a little modification from TCP Reno at the sender side, so DAA-TCP can be easily developed and the additional overheads are very low.

Key words: transmission control protocol (TCP); congestion control; TCP performance; DAA-TCP (dual AMID-based active TCP)

摘 要: TCP Reno 是当前 Internet 中流行的 TCP(transmission control protocol)版本, 为改善其性能, 提出一种 TCP Reno 的改进方案, 取名 DAA-TCP(dual AMID-based active TCP).DAA-TCP 融合了双重 AIMD(additive-increase multiplicative-decrease)算法, 添加了主动拥塞控制, 即在满足给定条件下, 可主动降低拥塞窗口, 即便此时网络并未发 生拥塞.仿真分析了 DAA-TCP 的性能,结果表明,与 TCP Reno 相比,使用 DAA-TCP 可提高吞吐量、减少重传报文、 降低报文重传率;DAA-TCP 也能与 TCP Reno 友好共存. 另外, DAA-TCP 可由 TCP Reno 发送方作较小修改得到. 因 此,DAA-TCP 容易实现,所增加的额外开销较少.

关键词: 传输控制协议(TCP);拥塞控制;TCP 性能;DAA-TCP

中图法分类号: TP393 文献标识码: A

* Supported by the Foundation of National Development and Reform Commission of China under Grant No.CNGI-04-4-2D (国家发改 委基金); the Natural Science Foundation Project of CQ CSTC of China under Grant No.2006BB2164 (重庆市科委自然学科基金)

Received 2006-07-06; Accepted 2007-04-03 
TCP 是目前 Internet 中广泛采用的传输层协议,其拥塞控制主要基于 $\operatorname{AIMD}(a, b)$ (additive-increase multiplicative-decrease)算法 ${ }^{[1-5]}$.流行的 TCP 版本,TCP Reno 取 $a=1, b=0.5$.TCP Reno 采用的是一种反应式的被 动拥塞控制模式,即发送方只有在检测出网络拥塞时才启动拥塞控制机制 ${ }^{[1,2]}$. 由于采用被动的方式进行拥塞控 制,致使网络拥塞次数较多,数据重传难以有效抑制.TCP Reno 的主要优点在于其良好的网络动态响应性, 即在 网络未拥塞时能够快速地使用网络中的有用资源, 在网络发生拥塞时能够急剧地降低其数据发送速率, 快速减 轻网络拥塞. 不过也正因为如此,其数据发送速率的波动性较大,资源利用率受到限制,因为稳态时, 其平均拥塞 窗口值约为最大拥塞窗口值的 $3 / 4$ 倍. 对于 $a, b$ 值较小的基于 $\operatorname{AIMD}(a, b)$ 的拥塞控制,稳态时, 其数据发送速率的 波动性较小,带宽利用率较高,因为其平均拥塞窗口值约为最大拥塞窗口值的 $(2-b) / 2$ 倍. 不过,其网络动态响应 性不好, 比如不能快速地使用网络中的有用资源, 在网络发生拥塞时不能快速地降低数据发送速率以缓解网络 拥塞.

关于主动拥塞控制方面的研究目前主要集中在路由器的队列管理方面,典型的研究成果为随机早期检测 RED (random early detection) 技术 ${ }^{[6]}$. 关于改进 TCP Reno 以添加主动拥塞控制的研究, 迄今尚未见诸报道. 除了 我们之前的工作 ${ }^{[7]}$ 以外, 也未见双重 AIMD 算法在 TCP 中的应用研究. 在 TCP Vegas ${ }^{[8]}$ 和 TCP Veno ${ }^{[9]}$ 中, 有主动 拥塞控制的思想,尽管其中未明确使用“主动拥塞控制”这一未语. 然而, 它们与 TCP Reno 在共享网络环境下的 公平性不好.

本文提出了一种 TCP Reno 的改进方案,取名 DAA-TCP(dual AMID-based active TCP).DAA-TCP 与文献[7] 中方案的共同点是在 TCP 中引入了双重 AIMD 算法.它们的不同之处在于:(1) DAA-TCP 中基于 AIMD $(1,0.5)$ 的拥塞控制采用的是被动拥塞控制模式,基于 $\operatorname{AIMD}(a, b)(a, b$ 取较小值)的拥塞控制采用的是主动拥塞控制模 式,即发送方可主动降低拥塞窗口(尽管此时网络并未发生拥塞),从而可以有效地避免拥塞, 大大地降低报文重 传.文献[7]中,基于 $\operatorname{AIMD}(1,0.5)$ 的拥塞控制和基于 $\operatorname{AIMD}(a, b)$ 的拥塞控制采用的均是被动拥塞控制模式;(2) 文 献[7]中,基于 $\operatorname{AIMD}(1,0.5)$ 的拥塞控制用于系统暂态,基于 $\operatorname{AIMD}(a, b)$ 的拥塞控制用于系统稳态,其难点在于二者 之间切换条件的判别, 难于实施, 会出现误判. 在 DAA-TCP 中, 二者间的切换条件易于实施, 不涉及系统暂态与稳 态条件的严格判别,基于 $\operatorname{AIMD}(1,0.5)$ 的拥塞控制和基于 $\operatorname{AIMD}(a, b)$ 的拥塞控制交替使用.

\section{DAA-TCP 拥塞控制}

DAA-TCP 拥塞控制融合了双重 AIMD 算法,即采用 $\operatorname{AIMD}(1,0.5)$ 和 $\operatorname{AIMD}(a, b)$, 其中, $0<a<1,0<b<0.5$. 考虑到 与 TCP Reno 的公平性,要求 $a=3 b /(2-b)^{[5]}$.于是,DAA-TCP 拥塞控制有两种模式:基于 $\operatorname{AIMD}(1,0.5)$ 的拥塞控制模 式和基于 $\operatorname{AIMD}(a, b)$ 的拥塞控制模式,分别简称为被动拥塞控制模式和主动拥塞控制模式.两种模式之间进行 切换时,拥塞窗口值的降低也考虑了与 TCP Reno 的公平性.

在满足以下 4 个条件之一时,由被动拥塞控制模式切换至主动拥塞控制模式: 条件 (1):发送方本次收到 3 个 重复应答时的拥塞窗口值,与上一次收到的 3 个重复应答时的拥塞窗口值的相对误差小于给定门限;条件(2): 条 件 (1)不满足,而发送方本次收到 3 个重复应答时的拥塞窗口值,大于上一次收到的 3 个重复应答时的拥塞窗口 值; 条件(3):拥塞避免阶段增加过程中的拥塞窗口值,大于发送方最近一次收到的 3 个重复应答时的拥塞窗口值, 且此两值之比大于给定门限; 条件(4):相邻两次收到 3 个重复应答间,收到的应答个数小于 3.

条件(1)满足时,可初步认为连接已经估测出了其允许的最大可用网络资源; 条件(2)和条件(3)主要为了避免 某连接竞用网络资源过快而抑制了其他连接(主要是此时采用主动拥塞控制模式的连接)对网络资源的竞用,从 而影响公平性.在仿真分析中我们发现存在这一现象,引入条件(2)和条件(3)后可以有效地改善公平性; 条件(4)的 引入主要是为了缓解短期连续拥塞对连接吞吐量的影响,也考虑了一个发送窗口内多个报文丢失的情况 (与 TCP NewReno 类似,只是处理方式不同).

注意条件(2)与条件(3)的差异:条件(2)是在网络拥塞时判断,而条件(3)是在网络未拥塞时判断.进入主动拥塞 控制模式后, 可主动降低拥塞窗口, 即便此时网络并未发生拥塞. 主动降低拥塞窗口的最大次数与切换条件有 关,在条件(1) 条件 (4)下切换至主动拥塞控制模式后, 主动降低拥塞窗口的最大次数分别为 3,2,1,1(在仿真分析 
中我们也试用了其他取值,根据仿真,这一取值是有效的).图 1 为 DAA-TCP 由被动拥塞控制模式切换至主动拥 塞控制模式拥塞窗口示意图.

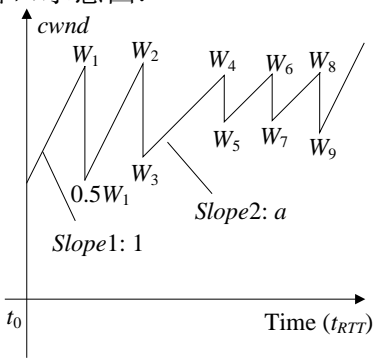

(a) Under condition(1

(a) 条件(1)满足

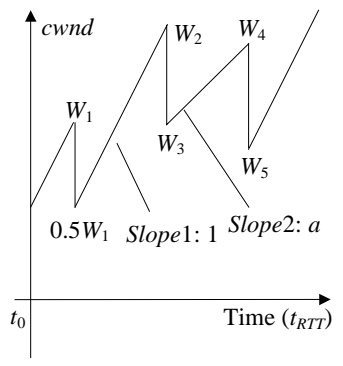

(c) Under condition(3) (c) 条件(3)满足

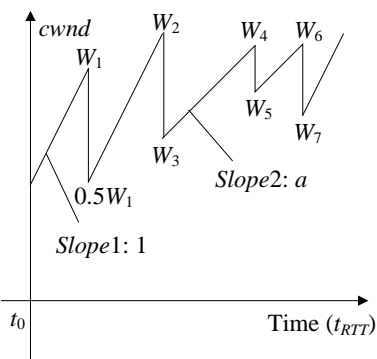

(b) Under condition(2) (b) 条件(2)满足

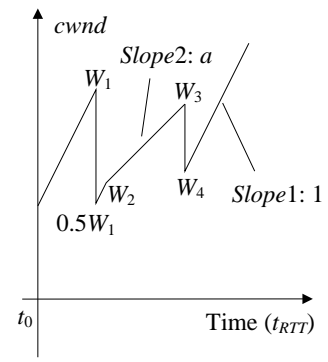

(d) Under condition(4) (d) 条件(4)满足

Fig.1 The cwnd from passive congestion control mode to active congestion control mode

图 1 由被动拥塞控制模式切换至主动拥塞控制模式 $c w n d$ 示意图

在满足以下两个条件之一时,由主动拥塞控制模式切换到被动拥塞控制模式:(I) 网络发生了拥塞,即发送 方收到了 3 个重复应答或超时;(II) 主动降低拥塞窗口次数,达到给定的最大值.此条件主要是为了让连接重新 快速地跟踪网络状态, 以便及时地响应网络条件的变化.在被动拥塞控制模式下,除了切换条件满足后会切换至 主动拥塞控制模式以外,其余操作与 TCP Reno 中相同.

因此,DAA-TCP 除了沿用 TCP Reno 中根据重复应答和超时来推断网络拥塞并降低拥塞窗口的被动拥塞 控制方式以外,还添加了主动降低拥塞窗口方式.下面结合图 1 详细说明,图中 $W_{i}(i=1,2,3, \ldots, 9)$ 为拥塞窗口值.

情形 1. $W_{1}$ 和 $W_{2}$ 为相邻两次收到 3 个重复应答时的拥塞窗口值.

1) 若 $\left[\left(W_{2}-W_{1}\right) / W_{1}\right]$ 的绝对值小于给定门限(即后述中的 $t h \_v c$ ), 则 $W_{3}=(1-b)[a /(2 b)] W_{2}$, 进入主动拥塞控 制模式.在此模式下,主动降低拥塞窗口的最大次数为 3,如图 1(a)所示.

a) 如果主动降低拥塞窗口次数能够达到最大值 3. 在 $W_{4} / W_{2}, W_{6} / W_{2}$ 和 $W_{8} / W_{2}$ 大于给定门限值(即后 述中的 $\left.t h \_c w\right)$ 时, 则主动降低拥塞窗口,相应的 $W_{5}=(1-b) W_{4}, W_{7}=(1-b) W_{6}, W_{9}=(b / a) W_{2}$, 之后切换 至被动拥塞控制模式;

b) 如果在主动降低拥塞窗口次数达到 3 之前,发送方检测出网络发生了拥塞,则立即切换至被动 拥塞控制模式.若收到了 3 个重复应答,则拥塞窗口值降低为当前值的 $(b / a)$ 倍;若超时,则按 TCP Reno 中的处理,即将拥塞窗口降为 1 , 进入慢启动阶段.

2) 若条件“ $\left[\left(W_{2}-W_{1}\right) / W_{1}\right]$ 的绝对值小于给定门限”不满足:

a) 如果 $W_{2}<W_{1}$, 则维持被动拥塞控制模式, $W_{3}=0.5 W_{2}$;

b) 如果 $W_{2}>W_{1}$, 则 $W_{3}=(1-b)[a /(2 b)] W_{2}$, 进入主动拥塞控制模式. 在此模式下,主动降低拥塞窗口的 最大次数为 2, 如图 1(b)所示. 后续过程与 1) 类似, 只是主动降低拥塞窗口的最大次数为 2 , 其 中, $W_{7}=(b / a) W_{2}$. 
3) 若 $W_{1}$ 和 $W_{2}$ 间收到的应答个数小于或等于 3 , 则不降低拥塞窗口,直接进入主动拥塞控制模式. 在此模 式下,主动降低拥塞窗口的最大次数为 1 , 如图 1(d)所示. 后续过程与 1 )类似,只是主动降低拥塞窗口的 最大次数为 1 , 其中 $W_{4}=(b / a) W_{1}$.

情形 $2 . W_{1}$ 为被动拥塞控制模式下在收到 3 个重复应答时的拥塞窗口值, $W_{2}$ 为此模式下拥塞避免阶段增加 过程中的拥塞窗口值.

1) 若 $W_{2} / W_{1}$ 大于给定门限(即后述中的 $t h \_l w$ ), 则主动降低拥塞窗口, $W_{3}=(1-b)[a /(2 b)] W_{2}$, 进入主动拥塞 控制模式. 在此模式下,主动降低拥塞窗口的最大次数为 1 ,如图 1(c)所示. 后续过程与情形 1 中的 1) 类似,只是主动降低拥塞窗口的最大次数为 1 , 其中 $W_{5}=(b / a) W_{2}$.

2) 若条件“ $W_{2} / W_{1}$ 大于给定门限”不满足,则可继续增大拥塞窗口.

\section{DAA-TCP 的 SDL 描述}

为了清晰地呈现 DAA-TCP, 图 2 给出了 DAA-TCP 核心功能的 SDL(specification and description language) 描述.DAA-TCP 的其他方面与 TCP Reno 中相同.

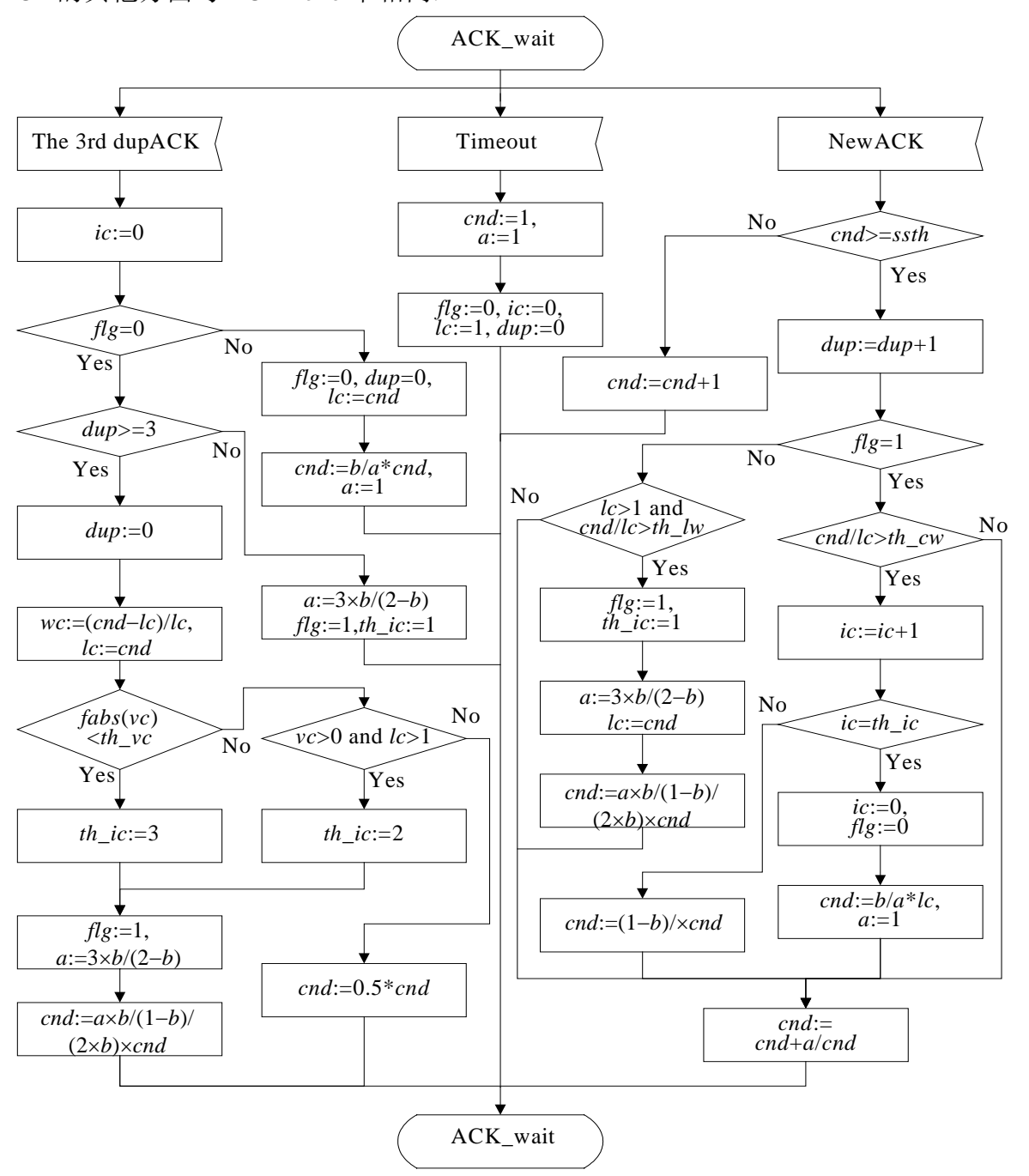

Fig.2 The SDL description of DAA-TCP core functions

图 2 DAA-TCP 核心功能的 SDL 描述 
图 2 的相关变量说明如下:

cnd:拥塞窗口值.

$a, b: A I M D(a, b)$ 中的加性因子和乘性因子.

$l c$ :最近一次收到 3 个重复应答时的拥塞窗口值(上节中情形 2 下 “收到 3 个重复应答但不降低拥塞口”时除 外), 或因拥塞窗口过大而主动降低拥塞窗口时的 $c n d\left(\right.$ 如图 1(b)中的 $W_{2}$ ). 初始值为 1 , 超时后复位为 1 . $v c$ :收到 3 个重复应答时的 $c n d$ 与 $l c$ 的相对误差.

$f l g$ :主动拥塞控制模式标志, flg $=1$ 时进入主动拥塞控制模式.此模式下,可主动降低拥塞窗口.初始值设为 0 ,

网络拥塞后复位为 0 .

$d u p:$ 拥塞避免阶段收到应答的个数.初始值为 0 ,网络拥塞后复位为 0 .

$i c$ :主动拥塞控制模式下,主动降低拥塞窗口的次数.初始值为 0 ,网络拥塞后复位为 0 .

$t h \_v c$ : 在 $v c$ 的绝对值小于此门限值时,进入主动拥塞控制模式,即令 $f l g=1$.

$t h \_c w$ :在主动拥塞控制模式下,若 $c n d / l c$ 大于此门限值,则主动降低拥塞窗口.

$t h \_l w$ : 在被动拥塞控制模式下,若 $c n d / l c$ 大于此门限值,则主动降低拥塞窗口,切换至主动拥塞控制模式.

th_ic:主动拥塞控制模式下,主动降低拥塞窗口次数的最大值.当 ic 达到此值时,切换至被动拥塞控制模式.

由以上描述可知,DAA-TCP 中有 4 个参数,即 $b, t h \_v c, t h \_c w$ 和 $t h \_l w$. 为考虑协议内公平性起见, 要求在所有 的 DAA-TCP 中使用一致的参数值.

\section{3 性能分析}

根据前述 DAA-TCP 的 SDL 描述, 在 ns-2.26 中实现 DAA-TCP.仿真中使用的拓扑如图 3 所示, 其中, $S_{1} \sim S_{10}$ 为发送方, $R_{1} \sim R_{10}$ 为接收方; $S_{\mathrm{CBR}}, R_{\mathrm{CBR}}$ 分别为背景流的发送方和接收方, 背景流为 $\mathrm{CBR}$. 在后续仿真结果中, 用 no-CBR 表示无背景流, 即 CBR 大小为 0 , 用 CBR 表示有背景流, 大小设为 $1 \mathrm{Mbps} . N_{1} \sim N_{4}$ 处的队列管理为 Drop-Tail, 缓冲大小为 40 个报文, 慢启动门限初始值设为 100.仿真工具采用 ns-2.26. 根据第 4 节中的仿真结果, DAA-TCP 中取 $b=0.06,3$ 个门限值分别设为 $t h \_v c=0.16$, $t h \_c w=0.94, t h \_l w=1.4$. 另外, 本节考察 DAA-TCP 与 TCP Reno 的比较,主要是因为:1) TCP Reno 是当前 Internet 中广泛采用的 TCP 版本;2) DAA-TCP 是基于 TCP Reno 的改进;3) DAA-TCP 能够与 TCP Reno 友好地共存, 它们之间的公平性较好;4) 其他方案虽然也有较好的性能, 但与 TCP Reno 的公平性不是很好.

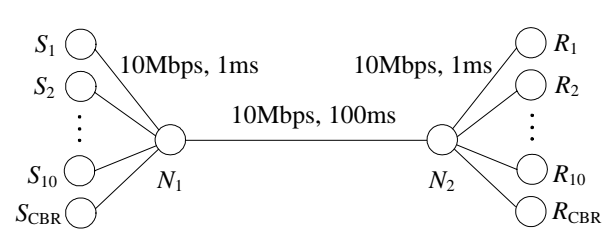

(a) Topology 1

(a) 拓扑 1

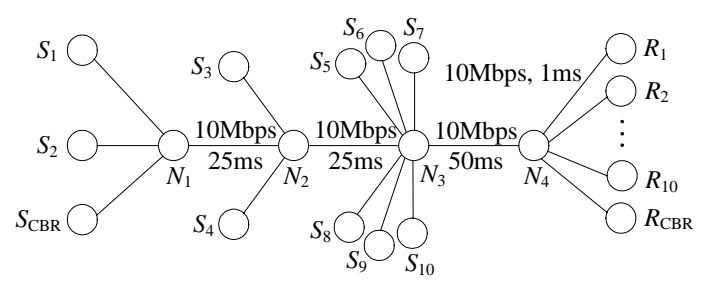

(b) Topology 2

Fig.3 Simulation topologies

图 3 仿真中使用的拓扑

仿真实验中考察的性能指标主要包括平均吞吐量、报文重传率和公平指数,其中,平均吞吐量和报文重传 率分别用符号 avgthrput 和 rexprob 表示.下面对仿真中考察的部分性能指标予以说明.

累计平均吞吐量:多个连接平均吞吐量之和. 比如,10 个连接的累计平均吞吐量为 10 个连接平均吞吐量之 和,用aggr_avgthrput 表示.

累计报文重传率:多个连接累计的报文重传率. 比如,10 个连接的累计报文重传率近似为 10 个连接从仿真 开始至给定时刻已经重传的报文总数除以 10 个连接已经传输的报文总数,用 aggr_rexprob 表示. 
吞吐量公平指数 $f_{i}$ 根据吞吐量计算的公平指数, 比如, 10 个连接吞吐量公平指数计算公式为

$$
\left(\Sigma T_{i}\right)^{2} /\left(10 \Sigma T_{i}^{2}\right),
$$

其中, $T_{i}$ 为连接 $i$ 的吞吐量. $f_{i}$ 可用于评价协议内公平性, 也可用于评价协议间的公平性.

\section{1 拓扑 1 下的性能}

拓扑 1 为简单亚铃拓扑,所有连接的网络环境相同. 在此拓扑下仿真 4 种场景,其中:场景 1 考察稳态性能; 场景 2 和场景 3 考察瞬态响应性, 即能否快速地使用网络中的有用资源, 当网络发生拥塞时, 能否快速地缓解网 络拥塞;场景 4 考察 DAA-TCP 与 TCP Reno 共存环境下的性能.

场景 1 . 所有连接均从 0 时刻开始,仿真时间为 1000 s.

图 4 图 6 分别为场景 1 下(所有连接为 DAA-TCP)无背景流时,DAA-TCP 的拥塞窗口 cwnd、平均吞吐量 avgthrput 和报文重传率 rexprob 随时间变化的曲线.

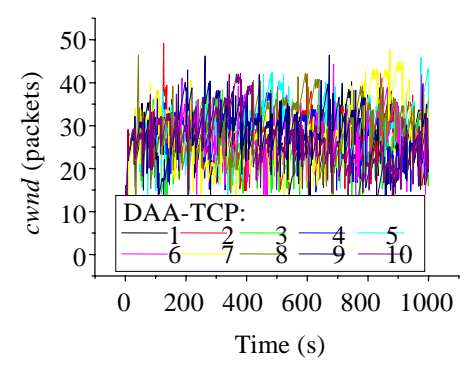

Fig.4 The cwnd of DAA-TCP for the scene 1

图 4 场景 1 下 DAA-TCP 的 cwnd

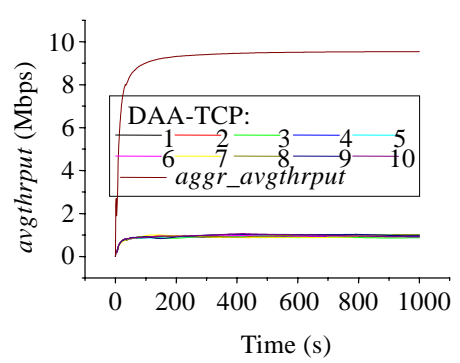

Fig.5 The avgthrput of DAA-TCP for the scene 1

图 5 场景 1 下 DAA-TCP 的 avgthrput

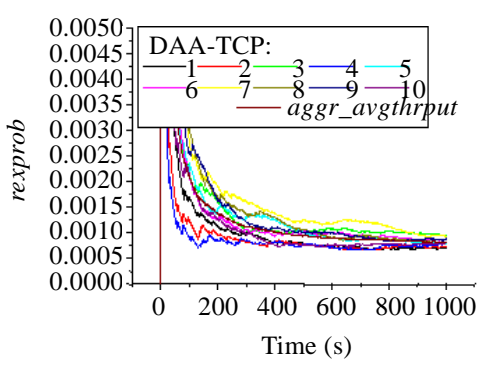

Fig.6 The rexprob of DAA-TCP for the scene 1

图 6 场景 1 下 DAA-TCP 的 rexprob

到 $1000 \mathrm{~s}$ 时,10 个 DAA-TCP 连接的吞吐量公平指数 $f_{i}=0.997811$, 说明 DAA-TCP 协议内公平性较好; 累计 平均吞吐量为 $9.54359 \mathrm{Mbps}$, 可认为链路利用率近似为 $95.44 \%$; 累计重传 983 个报文, 累计报文重传率为 $8.24008 \times 10^{-4}$.

场景 2. 所有连接均从 0 时刻开始,连接 8 10 在 500s 终止,仿真时间为 $1000 \mathrm{~s}$.

图 7 图 9 分别为场景 2 下(所有连接为 DAA-TCP)无背景流时,DAA-TCP 的拥塞窗口 cwnd、平均吞吐量 avgthrput 和报文重传率 rexprob 随时间的变化曲线.

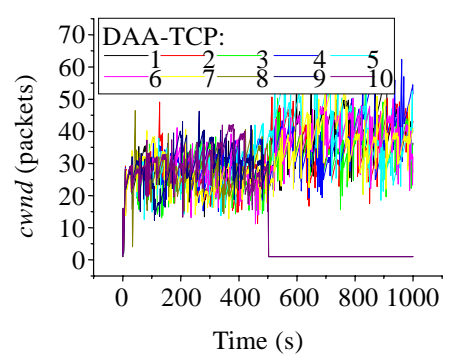

Fig.7 The cwnd of DAA-TCP for the scene 2

图 7 场景 2 下 DAA-TCP 的 cwnd

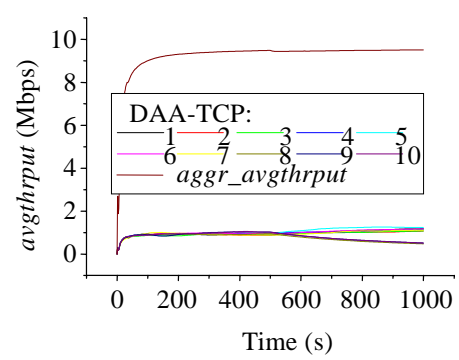

Fig.8 The avgthrput of DAA-TCP for the scene 2

图 8 场景 2 下 DAA-TCP 的 avgthrput

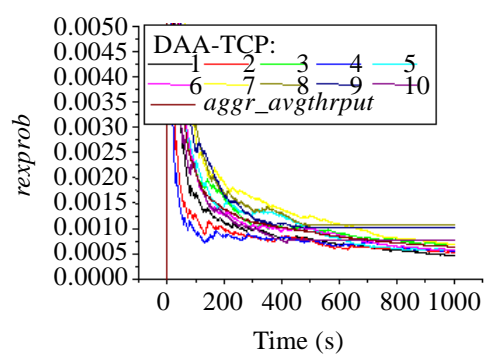

Fig.9 The rexprob of DAA-TCP for the scene 2

图 9 场景 2 下 DAA-TCP 的 rexprob

$500 \mathrm{~s}$ 后, 连接 1 7 快速争用因连接 8 10 终止而多出的可用资源, 其吞吐量增大; 连接 8 10 在终止后, 报文重 
传率维持不变,平均吞吐量随时间的推移逐渐下降.至 $1000 \mathrm{~s}$ 时,累计平均吞吐量为 $9.50522 \mathrm{Mbps}$, 累计重传 758 个报文,累计报文重传率为 $6.37965 \times 10^{-4}$.

场景 3. 连接 1 7 从 0 时刻开始,连接 8 10 从 500s 开始,仿真时间为 $1000 \mathrm{~s}$.

图 10 图 12 分别为场景 3 下(所有连接为 DAA-TCP)无背景流时,DAA-TCP 的拥塞窗口 cwnd、平均吞吐 量 avgthrput 和报文重传率 rexprob 随时间的变化曲线.

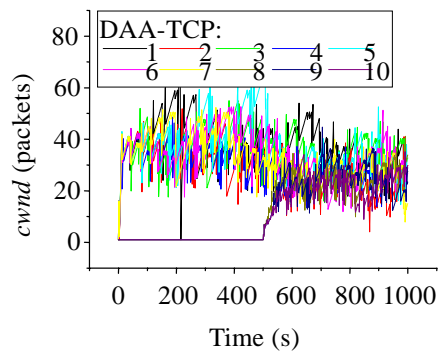

Fig.10 The $c w n d$ of DAA-TCP for the scene 3

图 10 场景 3 下 DAA-TCP 的 cwnd

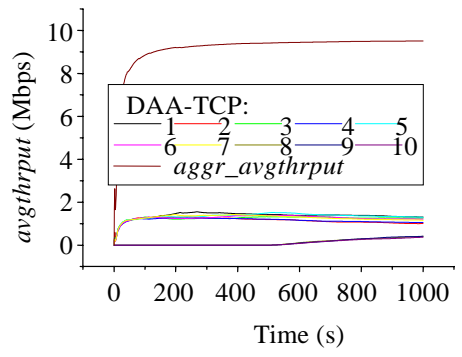

Fig.11 The avgthrput of DAA-TCP for the scene 3

图 11 场景 3 下 DAA-TCP 的 avgthrput

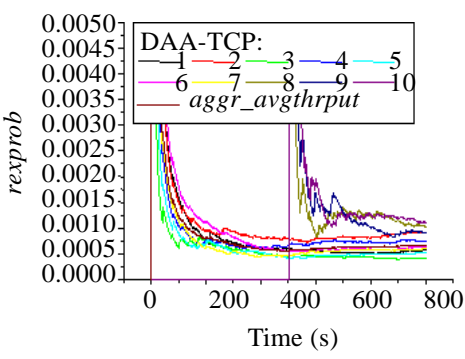

Fig.12 The rexprob of DAA-TCP

for the scene 3

图 12 场景 3 下 DAA-TCP 的 rexprob

500s 后,连接 1 7 快速降低数据发送速率,其吞吐量下降;连接 8 10 在 500s 开始后争用网络资源, 其吞吐量 增加.至 $1000 \mathrm{~s}$ 时,累计平均吞吐量为 $9.49911 \mathrm{Mbps}$, 累计重传 783 个报文, 累计报文重传率为 $6.5943 \times 10^{-4}$.

场景 2 和场景 3 的仿真结果说明,DAA-TCP 能够较好地适应网络环境的动态变化,累计吞吐量或链路利用 率始终维持较大值,报文重传率维持较低值.

为便于比较,仿真了场景 1 场景 3 下(所有连接为 TCP Reno)TCP Reno 的性能.表 1 归纳了 1 000s 时的累计 平均吞吐量、累计重传报文数及累计报文重传率, 其中,no-CBR 表示无背景流,CBR 表示有背景流, 大小为 $1 \mathrm{Mbps}$.

Table 1 Performance of DAA-TCP and TCP Reno for the scene 1 to scene 3

表 1 场景 1 场景 3 下 DAA-TCP 与 TCP Reno 的性能

\begin{tabular}{|c|c|c|c|c|c|}
\hline & & & $\begin{array}{l}\text { Aggregate average } \\
\text { throughput (Mbps) }\end{array}$ & $\begin{array}{l}\text { Number of aggregate } \\
\text { retransmitted packets }\end{array}$ & $\begin{array}{l}\text { Aggregate retransmit } \\
\text { probability }\left(\times 10^{-4}\right)\end{array}$ \\
\hline \multirow{3}{*}{ Scene 1} & no-CBR & $\begin{array}{l}\text { DAA-TCP } \\
\text { TCP Reno }\end{array}$ & $\begin{array}{l}9.54359 \\
8.02158\end{array}$ & $\begin{array}{c}983 \\
2970\end{array}$ & $\begin{array}{c}8.24008 \\
29.6\end{array}$ \\
\hline & גדים & DAA-TCP & 8.54595 & 1464 & 13.7 \\
\hline & CBR & TCP Reno & 7.42869 & 4021 & 43.3 \\
\hline \multirow{4}{*}{ Scene 2} & DP & DAA-TCP & 9.50522 & 758 & 6.37965 \\
\hline & |110-CBK| & TCP Reno & 8.01955 & 2285 & 22.8 \\
\hline & & DAA-TCP & 8.50066 & 1094 & 10.3 \\
\hline & CBR & TCP Reno & 7.32618 & 3182 & 34.7 \\
\hline \multirow{4}{*}{ Scene 3} & no-CBR & DAA-TCP & 9.49911 & 783 & 6.5943 \\
\hline & & TCP Reno & 7.9947 & 2348 & 23.5 \\
\hline & $C \mathrm{RP}$ & DAA-TCP & 8.48449 & 1097 & 10.3 \\
\hline & CBR & TCP Reno & 7.3055 & 2970 & 32.5 \\
\hline
\end{tabular}

表 1 表明,在相同网络环境下,与 TCP Reno 相比,DAA-TCP 性能改善显著,即 DAA-TCP 的累计平均吞吐量 大幅度提高,累计重传报文极大地减小,累计报文重传率大为降低.例如,就场景 1 (无背景流)而言,到 $1000 \mathrm{~s}$ 时, TCP Reno 的累计平均吞吐量和累计报文重传率分别为 8.021 58Mbps 和 0.002 96;DAA-TCP 的累计平均吞吐量 和累计报文重传率分别为 9.54359Mbps 和 0.000824. 从而相对于 TCP Reno, 到 $1000 \mathrm{~s}$ 时,DAA-TCP 的累计平均 吞吐量提高了(9.54359-8.02158)/8.02158=18.9739\%, 累计报文重传率下降了 $|(0.000824-0.00296) / 0.00296|=$ $72.162 \%$. 
场景 4. 所有连接均从 0 时刻开始, 其中,连接 1 n 采用 DAA-TCP,连接 $n+1 \sim 10$ 采用 TCP Reno, 仿真时间为 1000 s.

图 13 图 15 分别为场景 4 下 $(n=5)$ 无背景流时,DAA-TCP 和 TCP Reno 的拥塞窗口 $c w n d$ 、平均吞吐量 avgthrput 和报文重传率 rexprob 随时间的变化曲线.

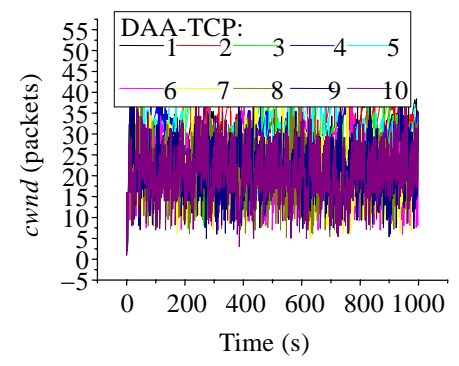

Fig.13 The $c$ wnd for the scene 4

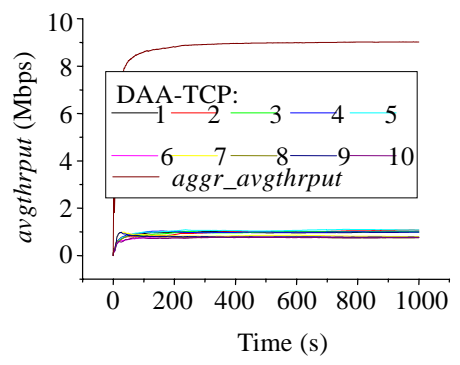

Fig.14 The avgthrput for the scene 4 图 14 场景 4 下的 avgthrput

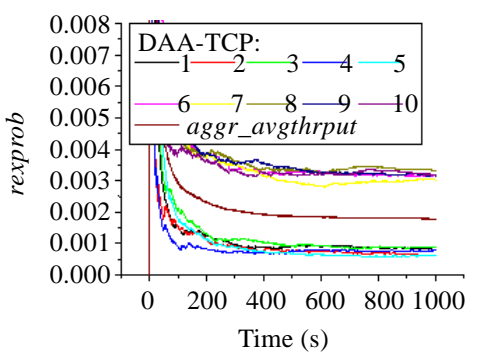

Fig.15 The rexprob for the scene 4

图 15 场景 4 下的 rexprob

至 $1000 \mathrm{~s}$ 时, 吞吐量公平指数 $f_{i}=0.978938$, 累计平均吞吐量为 $9.02505 \mathrm{Mbps}$, 累计重传 2009 个报文,累计报 文重传率为 0.00178. 改变 DAA-TCP 与 TCP Reno 连接的比例, 重复此仿真. 表 2 和表 3 归纳了仿真结果,表中 DAA-TCP/TCP Reno 表示 DAA-TCP 与 TCP Reno 连接个数的比例,如 DAA-TCP/TCP Reno=2/8 表明有 2 个 DAA-TCP 连接、 8 个 TCP Reno 连接.

Table 2 Performance for the environment with DAA-TCP and TCP Reno (no-CBR)

表 2 DAA-TCP 与 TCP Reno 共存环境下的性能(无背景流)

\begin{tabular}{|c|c|c|c|c|c|c|c|c|c|c|c|}
\hline DAA-TCP/TCP Reno & $0 / 10$ & $1 / 9$ & $2 / 8$ & $3 / 7$ & $4 / 6$ & $5 / 5$ & $6 / 4$ & $7 / 3$ & $8 / 2$ & $9 / 1$ & $10 / 0$ \\
\hline Aggregate average throughput & 8.0216 & 8.2647 & 8.485 & 8.6697 & 8.8323 & 9.0251 & 9.1607 & 9.2962 & 9.4297 & 9.501 & 9.5436 \\
\hline Number o & 2970 & 2747 & 2575 & 2379 & 2206 & 2009 & 1811 & 1638 & 1422 & 1196 & 983 \\
\hline Aggregate retransmit probability $\left(\times 10^{-3}\right)$ & 2.96 & 2.66 & 2.43 & 2.2 & 2.0 & 1.78 & 1.58 & 1.41 & 1.21 & 1.01 & 0.824 \\
\hline Fairness index $f_{i}$ & 0.9998 & .991 & 0.980 & 0.978 & .975 & 0.978 & 0.9830 & 0.985 & .987 & 0.9950 & 0.9978 \\
\hline
\end{tabular}

Table 3 Performance for the environment with DAA-TCP and TCP Reno (CBR)

表 3 DAA-TCP 与 TCP Reno 共存环境下的性能(有背景流)

\begin{tabular}{|c|c|c|c|c|c|c|c|c|c|c|c|}
\hline DAA-TCP/TCP Reno & $0 / 10$ & $1 / 9$ & $2 / 8$ & $3 / 7$ & $4 / 6$ & $5 / 5$ & $6 / 4$ & $7 / 3$ & $8 / 2$ & $9 / 1$ & $10 / 0$ \\
\hline sate average th & 7.4287 & 7.5310 & 7.6514 & 7.7526 & 7.8582 & 7.9584 & 8.1009 & 8.2158 & 8.3677 & 8.4573 & 8.5460 \\
\hline Numl & 4021 & 3638 & 3379 & 3056 & 2921 & 2610 & 2405 & 2041 & 1782 & 1609 & 1464 \\
\hline Aggregate retro & 4.33 & 3.86 & 3.53 & 3.15 & 2.97 & 2.62 & 2.38 & 1.99 & 1.7 & 1.52 & 1.37 \\
\hline Fai & 0.999 & 095 & 0.992 & 0.993 & 0.985 & .981 & .986 & 0.984 & 0.9829 & 0.987 & 0.9952 \\
\hline
\end{tabular}

表 2 和表 3 的结果表明,DAA-TCP 可与 TCP Reno 友好共存,公平地竞争网络资源; 在 TCP Reno 环境中加 入 DAA-TCP, 能够改善平均吞吐量,减少重传报文,降低报文重传率.随着 DAA-TCP 连接与 TCP Reno 连接比例 的增大,累计平均吞吐量,累计报文重传率下降.

\section{2 拓扑 2 下的性能}

拓扑 2 有 3 跳主干链路,部分连接的网络环境存在差异,相对于拓扑 1 而言,此拓扑要复杂一些.在此拓扑下 仿真 2 种场景,其中,场景 5 考察稳态性能,场景 6 考察 DAA-TCP 与 TCP Reno 的共存性能.

场景 5. 所有连接均从 0 时刻开始,仿真时间为 $1000 \mathrm{~s}$.

图 16 图 18 分别为场景 5 下(所有连接为 DAA-TCP)有背景流时,DAA-TCP 的拥塞窗口 $c w n d$ 、平均吞吐 量 avgthrput 和报文重传率 rexprob 随时间的变化曲线. 


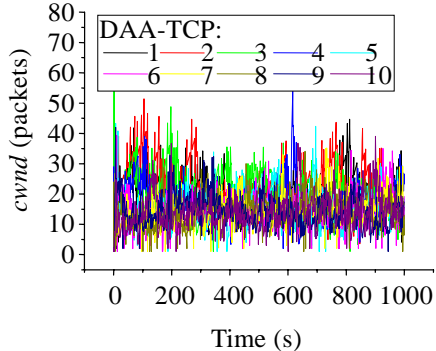

Fig.16 The $c$ wnd of DAA-TCP for the scene 5

图 16 场景 5 下 DAA-TCP 的 cwnd

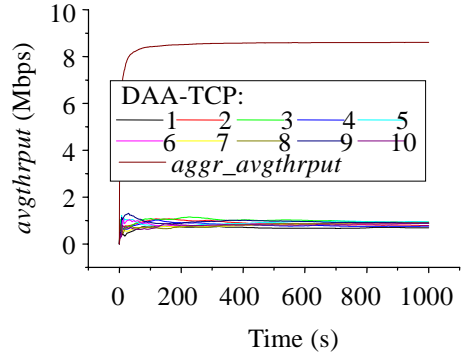

Fig.17 The avgthrput of DAA-TCP for the scene 5

图 17 场景 5 下 DAA-TCP 的 avgthrput

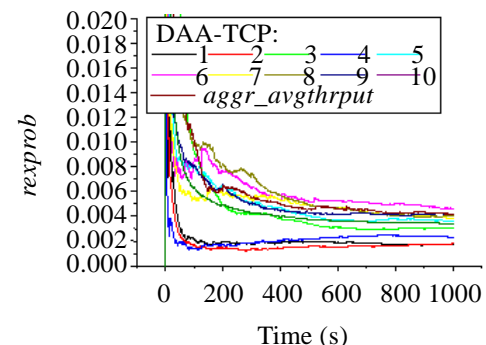

Fig.18 The rexprob of DAA-TCP for the scene 5

图 18 场景 5 下 DAA-TCP 的 rexprob

到 1 000s 时,10 个 DAA-TCP 连接的累计平均吞吐量为 8.606 86Mbps, 可以认为,第 3 跳主干链路的带宽利 用率近似为 $96.1 \%$ (有 $1 \mathrm{Mbps}$ 的背景流), 累计重传 3669 个报文,累计报文重传率为 0.00341 .

为便于比较,也仿真了场景 5 下(所有连接为 TCP Reno)有背景流时,TCP Reno 的性能. 图 19 图 21 分别为 TCP Reno 的拥塞窗口 cwnd、平均吞吐量 avgthrput 和报文重传率 rexprob 随时间的变化曲线.

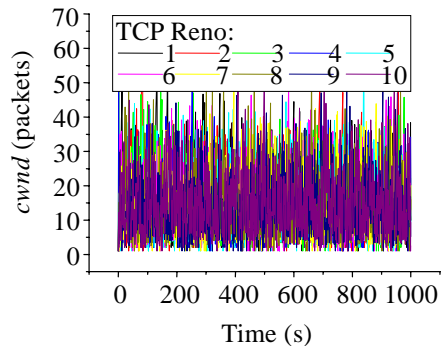

Fig.19 The cwnd of TCP Reno for the scene 5

图 19 场景 5 下 TCP Reno 的 cwnd

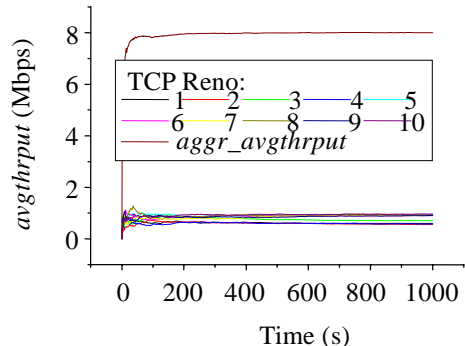

Fig.20 The avgthrput of TCP Reno

for the scene 5

图 20 场景 5 下 TCP Reno 的 avgthrput

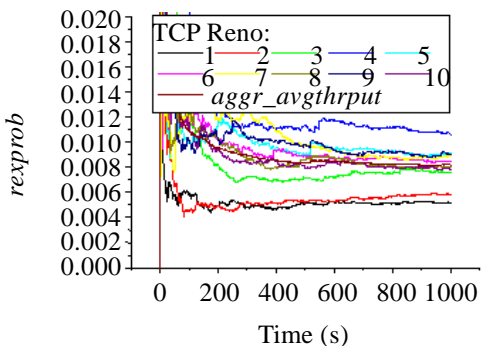

Fig.21 The rexprob of TCP Reno for the scene 5

图 21 场景 5 下 TCP Reno 的 rexprob

到 1 000s 时,10 个 TCP Reno 连接的累计平均吞吐量为 7.99886Mbps, 可以认为,第 3 跳主干链路的带宽利 用率近似为 89.99\%(因有 1Mbps 的背景流),累计重传 8192 个报文,累计报文重传率为 0.00819 .

从而就场景 5 而言,相对于 TCP Reno,到 1000 秒时,DAA-TCP 的累计平均吞吐量提高了 7.6\%,累计报文重 传率下降了 $58.36 \%$.

场景 6. 所有连接均从 0 时刻开始,其中,连接 1 4、连接 8 10 采用 TCP,连接 5 7 采用 DAA-TCP,仿真时间 为 $1000 \mathrm{~s}$.

图 22 图 24 分别为场景 6 下有背景流时,DAA-TCP 和 TCP Reno 的拥塞窗口 cwnd、平均吞吐量 avgthrput 和报文重传率 rexprob 随时间的变化曲线.

到 1 000s 时,累计平均吞吐量为 8.208 58Mbps, 累计重传 6803 个报文,累计报文重传率为 0.00663 ,连接 5 10 的吞吐量公平指数 $f_{i}=0.990749$. 因此,DAA-TCP 可与 TCP Reno 友好共存,DAA-TCP 的引入提高了平均吞吐量, 减少了重传报文,降低了报文重传率.

场景 5 和场景 6 的仿真结果说明,在较为复杂的网络环境下引入 DAA-TCP, 同样能够提高网络资源利用率, 减少重传报文,降低报文重传率,从而提高传输的效率;DAA-TCP 也能在复杂网络环境下与 TCP Reno 友好共存. 


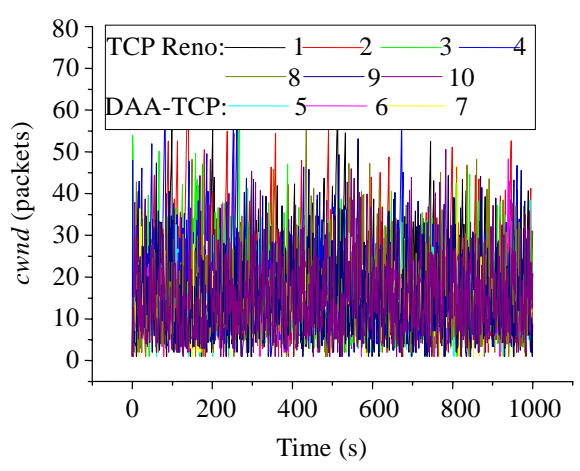

Fig.22 The cwnd for the scene 6

图 22 场景 6 下的 cwnd

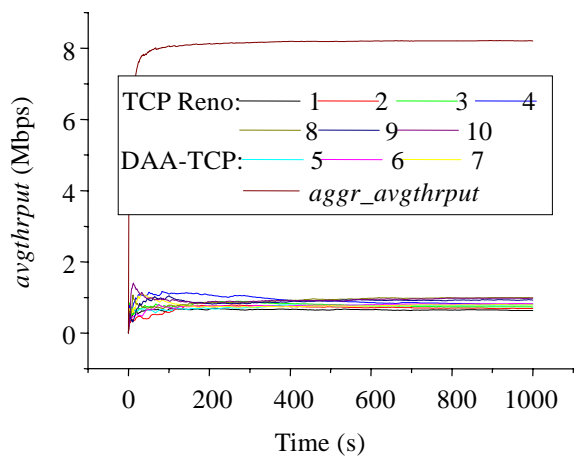

Fig.23 The avgthrput for the scene 6 图 23 场景 6 下 avgthrput

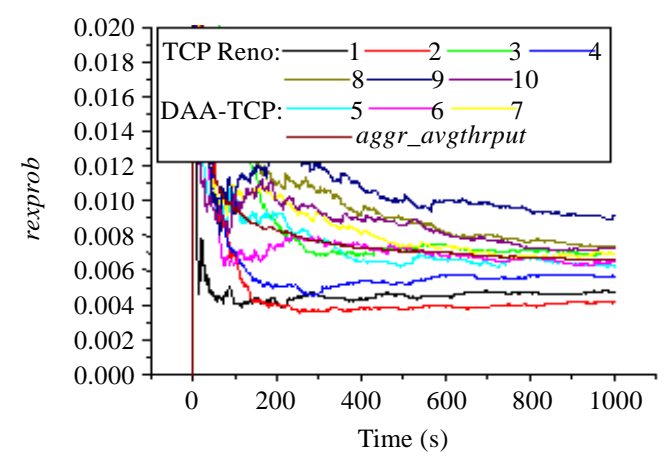

Fig.24 The rexprob for the scene 6

图 24 场景 6 下的 rexprob

\section{DAA-TCP 中的参数}

DAA-TCP 中有 4 个参数, 即 $b, t h \_v c, t h \_c w$ 和 $t h \_l w$. 为考察它们对 DAA-TCP 性能的影响,改变它们的取值, 重复场景 1 的仿真.仿真结果如图 25 图 28 所示.

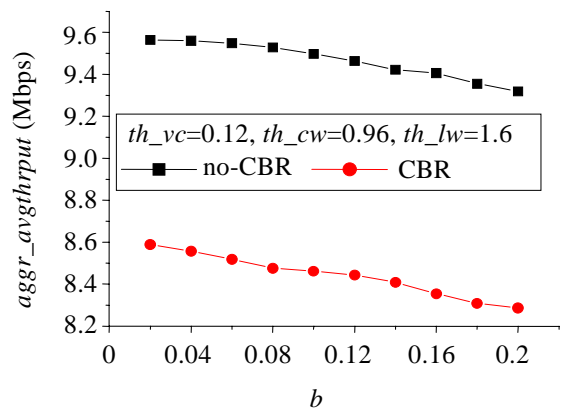

(a) aggr_avgthrput

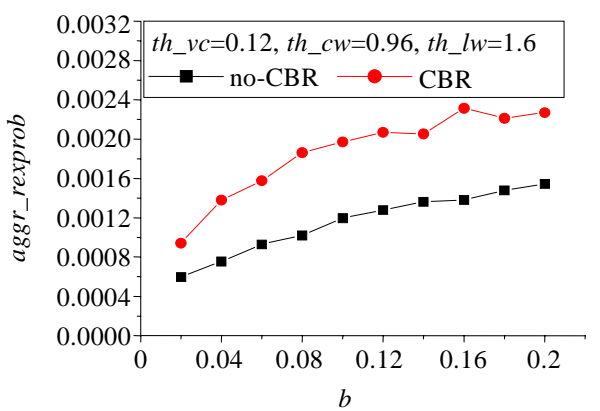

(b) aggr_rexprob

Fig.25 The aggr_avgthrput and aggr_rexprob vs. $b$

图 25 aggr_avgthrput 和 aggr_rexprob 随 $b$ 的变化 


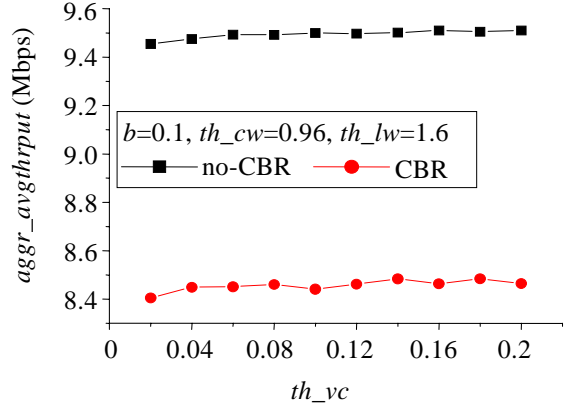

(a) aggr_avgthrput

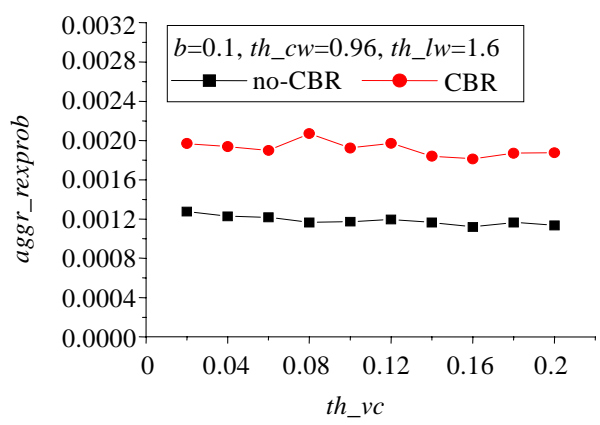

(b) aggr_rexprob

Fig.26 The aggr_avgthrput and aggr_rexprob vs. th_vc

图 26 aggr_avgthrput 和 aggr_rexprob 随 th_vc 的变化

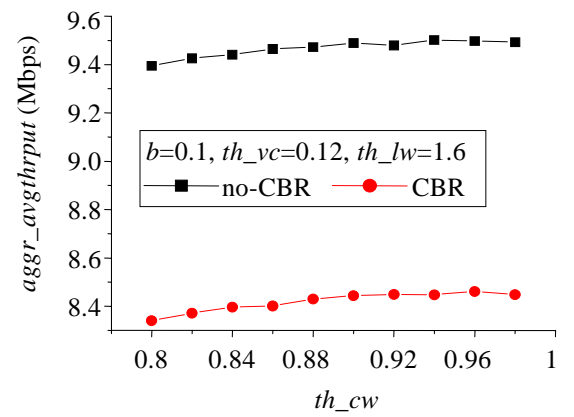

(a) aggr_avgthrput

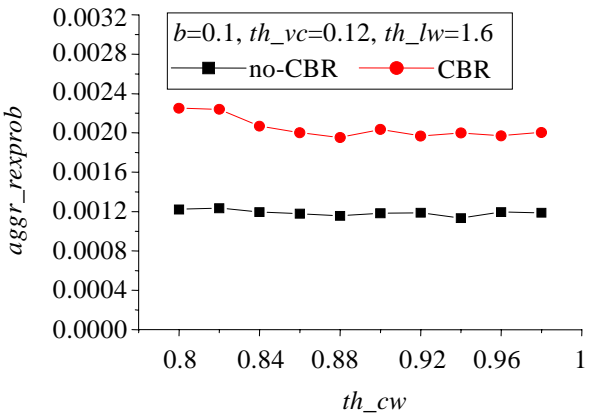

(b) aggr_rexprob

Fig.27 The aggr_avgthrput and aggr_rexprob vs. th_cw

图 27 aggr_avgthrput 和aggr_rexprob 随 $t h \_c w$ 的变化

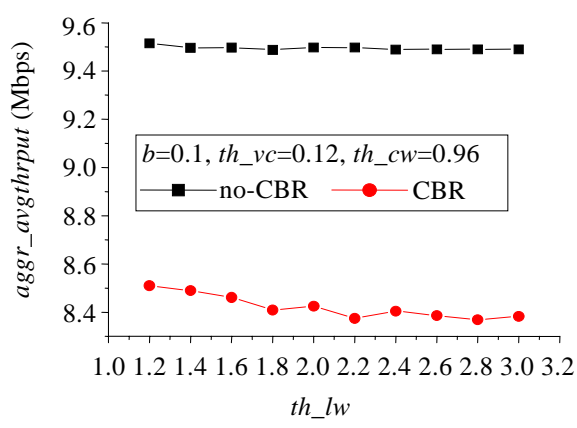

(a) aggr_avgthrput

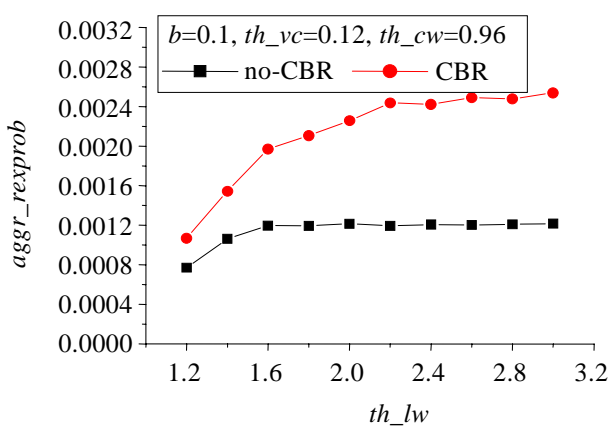

(b) aggr_rexprob

Fig.28 The aggr_avgthrput and aggr_rexprob vs. th_lw

图 28 aggr_avgthrput 和 aggr_rexprob 随 th_lw 的变化

由图 25 图 28 结果易知,DAA-TCP 中的 4 个参数均会影响 DAA-TCP 性能,其中, $b$ 是影响 DAA-TCP 性能 的主要参数.如较小的 $b$ 和 $t h \_l w$ 或者较大的 $t h \_v c$ 和 $t h \_c w$ 均可提高吞吐量,降低报文重传率.

不过,鉴于 TCP Reno 是当前 Internet 中广泛使用的 TCP 版本, 因此, 在参数选取方面要考虑 DAA-TCP 与 TCP Reno 的公平性. 图 29 图 32 给出了场景 4 下 $(n=5)$ 公平指数 $f_{i}$ 分别随 $b, t h \_v c, t h \_c w$ 和 $t h \_l w$ 的变化曲线. 


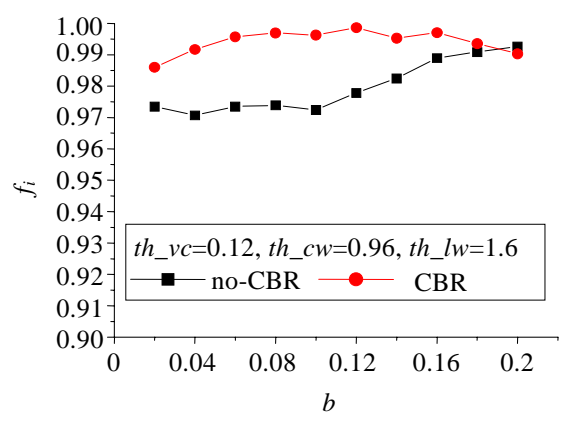

Fig.29 The $f_{i}$ vs. $b$

图 $29 f_{i}$ 随 $b$ 的变化

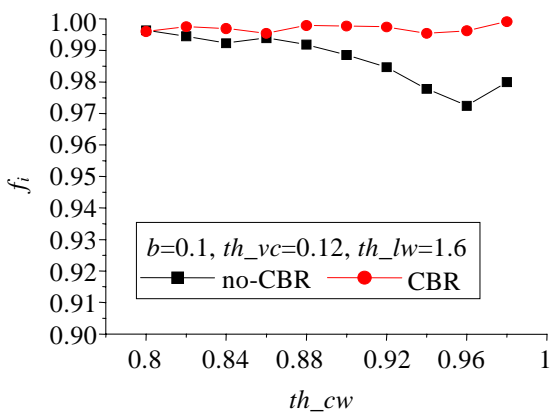

Fig.31 The $f_{i}$ vs. th_cw

图 $31 f_{i}$ 随 th_cw 的变化

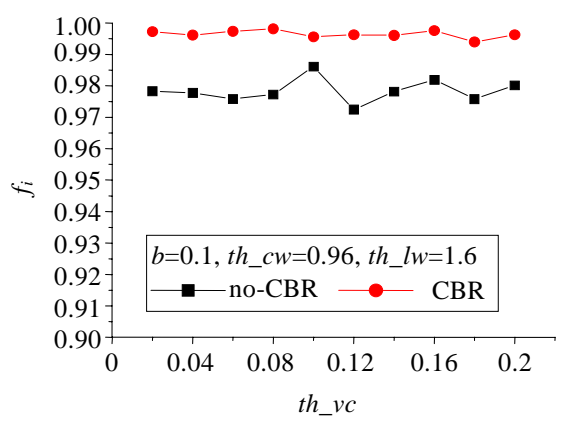

Fig.30 The $f_{i}$ vs. th_vc 图 $30 f_{i}$ 随 th_vc 的变化

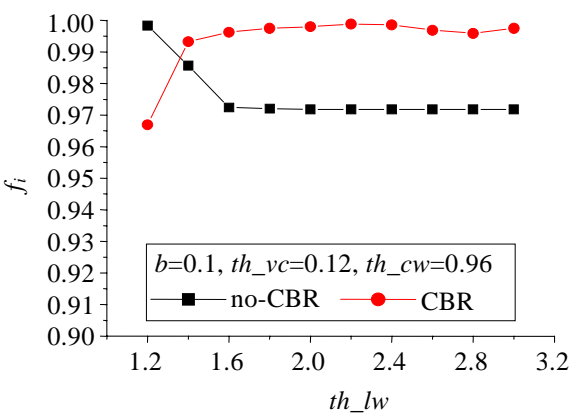

Fig.32 The $f_{i}$ vs. th_lw

图 $32 f_{i}$ 随 $t h \_l w$ 的变化

结果表明,对 DAA-TCP 与 TCP Reno 协议间公平性影响较大的参数是 $b$ 和 $t h \_c w$, 如增大 $b$ 或减小 $t h \_c w$ 均可显著改善 DAA-TCP 与 TCP Reno 协议间公平性; 较小的 $t h \_l w$ 也能有效改善 DAA-TCP 与 TCP Reno 协议 间公平性; th_vc 对 DAA-TCP 与 TCP Reno 公平性影响不大.

本节中给出的仿真结果为这些参数的选取提供了一定的参考. 这些参数在实际环境下的取值还需要结合 实际网络环境做进一步的探讨.

\section{5 结束语}

本文的主要贡献是提出了在 TCP Reno 中添加主动拥塞控制的思想,融合了双重 AIMD算法,给出了一种改 进的 TCP Reno 方案,取名 DAA-TCP. 结果表明,使用 DAA-TCP 可改善吞吐量,提高网络资源利用率,减少重传报 文,降低报文重传率;DAA-TCP 可与 TCP Reno 友好共存,公平竞争网络资源;DAA-TCP 可由 TCP Reno 发送方较 小修改而得, 因此易于实现,所增加的额外开销小.鉴于 TCP Reno 是当前 Internet 中广泛采用的 TCP 版本, 而 DAA-TCP 是基于 TCP Reno 的改进,且能与 TCP Reno 友好地共存,因此,DAA-TCP 具有良好的可部署性.

另外,我们也作了 DAA-TCP 与文献[7]中方案的性能分析比较(限于篇幅,未给出详细仿真结果), 结果表 明:(1) 二者在吞吐量以及与 TCP Reno 的公平性这两方面的性能基本一致;(2) 在报文重传率方面, DAA-TCP 比文献[7]中的方案要改善很多,这主要是因为在 DAA-TCP 中引入了主动拥塞控制机制.

本方所提出的方案对于无线网络中的移动终端尤其重要, 因为移动终端是靠电池供电的, 采用 DAA-TCP 可提高传输的效率,减少不必要的电能消耗.

致谢 在此,我们向对本文工作给予支持和建议的同行表示感谢. 


\section{References:}

[1] Stevens WR. TCP slow start: Congestion avoidance, fast retransmit, and fast recovery algorithms. RFC2001, 1997.

[2] Allman M, Paxson V, Stevens WR. TCP congestion control. RFC2581, 1999.

[3] Chiu DM, Jain R. Analysis of the increase and decrease algorithms for congestion avoidance in computer networks. Computer Networks and ISDN Systems, 1989,17(1):1-14.

[4] Floyd S. Connections with multiple congestioned gateways in packet-switched networks, part 1: One-way traffic. ACM Computer Communication Review, 1991,21(5):30-47.

[5] Floyd S, Handley M, Padhye J, Widmer J. Equation-Based congestion control for unicast applications: The extended version. Technical Report, TR-00-03, 2000. http://www.aciri.org/tfrc/

[6] Floyd S, Jacobson V. Random early detection gateways for congestion avoidance. IEEE/ACM Trans. on Networking, 1993,1(4): 397-413.

[7] Xu CB, Long KP, Yang SZ. Dual AIMD-based TCP congestion control. Journal of Computer Research and Development, 2003, 40(8):1175-1180 (in Chinese with English abstract).

[8] Brakmo LS, Peterson LL. TCP Vegas: End to end congestion avoidance on a global Internet. IEEE Journal on Selected Areas Communications, 1995,13(8):1465-1480.

[9] Fu CP, Liew SC. TCP Veno: TCP enhancement for transmission over wireless access networks. IEEE JSAC, 2003,21(2):216-228.

附中文参考文献:

[7]徐昌彪,隆克平,杨士中.基于双重 AIMD 的 TCP 拥塞控制.计算机研究与发展, 2003,40(8):1175-1180.

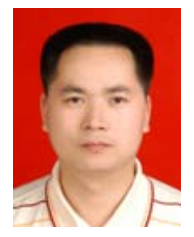

徐昌彪(1972-),男,贵州思南人,博士,副 教授,主要研究领域为 TCP/IP 性能分析与 评估,网络拥塞控制,宽带无线接入.

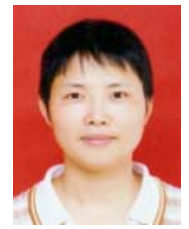

为网络管理与测量,网络安全.

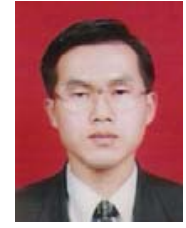

唐朝伟(1966-),男,博士,副研究员,主要 研究领域为宽带无线接入.

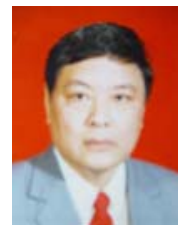

杨士中 $(1937-)$, 男, 教授,博士生导师,中 国工程院院士,主要研究领域为测控及遥 感信息传输. 